is the first demonstration of line-specific memory in individual invertebrates. In a study based on only two bacterial strains, maternally transferred immunity seemed to be strain-specific ${ }^{9}$. Invertebrates are occasionally capable of a specific response in the context of allogenic recognition ${ }^{10}$, but specificity in these cases is based on compatibility factors that have evolved within the species and so is not directly comparable with defence against a parasite.

The mechanism responsible for the linespecific memory in this copepod remains to be investigated, although binding by lectins is one possibility ${ }^{11}$. If specific memory in invertebrate defence is more widespread than originally thought, it would have implications for our understanding of innate immunity, host-parasite coevolution, and invertebrate-vector diseases such as malaria. Joachim Kurtz, Karoline Franz

Department of Evolutionary Ecology, Max Planck Institute of Limnology, August-Thienemann Strasse 2, 24306 Plön, Germany

email:kurtz@mpil-ploen.mpg.de

1. Janeway, C. A., Travers, P., Walport, M. \& Capra, J. D. Immunobiology: The Immune System in Health and Disease (Current Biology, London, 1999).

2. Hoffmann, J. A. \& Reichhart, J. M. Nature Immunol. 3, 121-126 (2002).

3. Klein, J. Scand. J. Immunol. 29, 499-505 (1989).

4. Schmid-Hempel, P. \& Ebert, D. Trends Ecol. Evol. 18, 27-32 (2003).

5. Rolff, J. \& Siva-Jothy, M. T. Science 301, 472-475 (2003).

6. Faulhaber, L. M. \& Karp, R. D. Immunology 75, 378-381 (1992).

7. Carius, H. J., Little, T. J. \& Ebert, D. Evolution 55, 1136-1145 (2001).

8. Kurtz, J., van der Veen, I. T. \& Christen, M. Exp. Parasitol. 100, 36-43 (2002).

9. Little, T. J., O'Connor, B., Colegrave, N., Watt, K. \& Read, A. F. Curr. Biol. 13, 489-492 (2003).

10. Scofield, V. L., Schlumpberger, J. M., West, L. A. \& Weissman, I. L. Nature 295, 499-502 (1982).

11. Marques, M. R. F. \& Barracco, M. A. Aquaculture 191, 23-44 (2000).

Competing financial interests: declared none.

\section{Microfluidic systems}

\section{High radial acceleration in microvortices}

icrofluidic systems can conveniently be used for rapid analysis of biological samples ${ }^{1-5}$. Here we describe a single re-circulating flow, or microvortex, that can generate a maximum fluid rotational velocity of up to $12 \mathrm{~m} \mathrm{~s}^{-1}$ and a corresponding radial acceleration in excess of $10^{6} \mathrm{~g}$. Such microvortices may be exploited in centrifugal microdevices to investigate the effects of high radial acceleration on biological and chemical processes.

A microvortex was generated in a diamond-shaped microchamber (Fig. 1a), which was visualized by using fluorescent polystyrene beads (diameter, $2 \mu \mathrm{m}$; Fig. $1 \mathrm{~b}$, left). The average linear velocity of the pressure-driven flow in the 30 - $\mu \mathrm{m}$-wide channel was $3 \mathrm{~m} \mathrm{~s}^{-1}$. The diamond-shaped notch along the side wall of an otherwise smooth channel causes flow detachment at the opening of the notch.

Unlike macroscale lid-driven cavity flow ${ }^{6}$, the combination of extremely tight rotation radii $(r<50 \mu \mathrm{m})$ and high rotational velocity $\left(v>10 \mathrm{~m} \mathrm{~s}^{-1}\right)$ of fluid flow from constricted microchannels can lead to generation of radial accelerations $\left(v^{2} / r\right)$ as high as $10^{7} \mathrm{~m} \mathrm{~s}^{-2}$. As the average linear velocity in the $30-\mu \mathrm{m}$-wide channel was increased from 3 to $45 \mathrm{~m} \mathrm{~s}^{-1}$ (Reynolds number $(\mathrm{Re})=245$ at the microchamber opening), the polystyrene beads (density, $\rho=1.05 \mathrm{~g} \mathrm{~cm}^{-3}$ ) in water were spun out of the microchamber owing to high centrifugal acceleration (Fig. 1b, right).

To illustrate the potential applications of this microvortex system, we flowed red polystyrene beads $\left(\rho=1.05 \mathrm{~g} \mathrm{~cm}^{-3}\right)$ and green silica beads $\left(\rho, 1.8-2.0 \mathrm{~g} \mathrm{~cm}^{-3}\right)$ in a solution of $50 \%(w / w)$ of $\mathrm{CsCl}\left(\rho=1.56 \mathrm{~g} \mathrm{~cm}^{-3}\right.$;
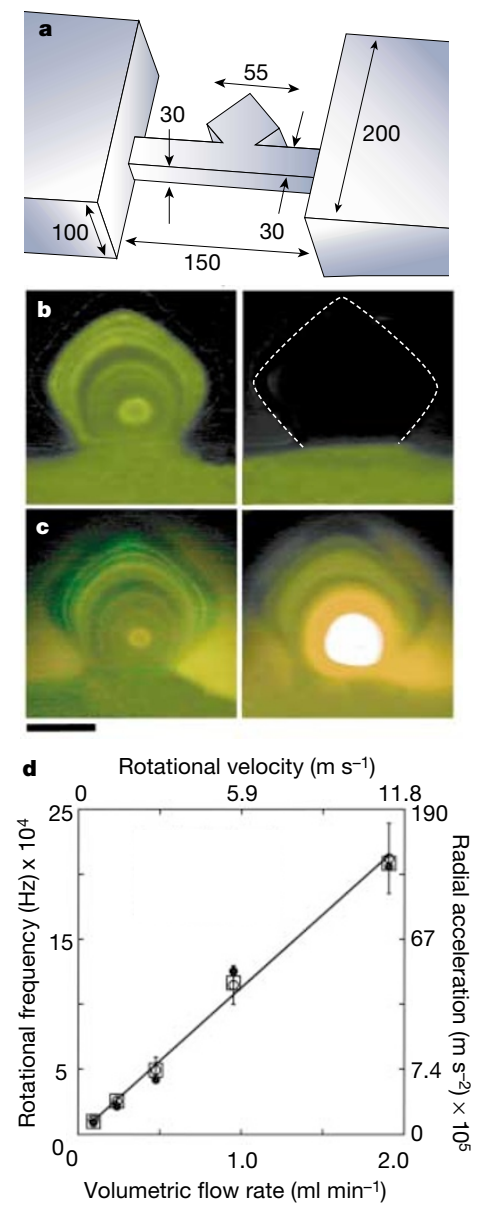

Figure 1 Generation and application of microvortices. a, Microchannel used to create the microvortex shown in $\mathbf{b}, \mathbf{c}$; dimensions are in $\mu \mathrm{m}$. $\mathbf{b}$, Fluorescent beads are spun out of the chamber as the flow velocity increases (from left to right). c, Separation of low-density red beads from high-density green beads in the microvortex as the flow velocity increases (from left to right). Scale bar, $25 \mu \mathrm{m}$. d, The experimentally measured rotational rates (squares and open circles) show excellent agreement with the vorticity results (filled circles) extracted from our simulations (some symbols overlap).
Fig. 1c). As the average flow velocity in the $30-\mu \mathrm{m}$-wide channel was increased from $1.5 \mathrm{~m} \mathrm{~s}^{-1}(\mathrm{Re}=13$ at the cavity's opening) to $20 \mathrm{~m} \mathrm{~s}^{-1}$ ( $\mathrm{Re}=122$ at the cavity's opening), the red fluorescent beads were concentrated towards the centre of the vortex, whereas the green beads were spun towards the chamber's outer edge. Although the linear flow velocity was $20 \mathrm{~m} \mathrm{~s}^{-1}$, the corresponding volumetric flow rate was only about $18 \mu \mathrm{s} \mathrm{s}^{-1}$, owing to the small cross-sectional area of the $30-\mu \mathrm{m}$ wide channel.

A simple calculation of the radial acceleration from the average flow rates in the microchannel overestimates the acceleration that we observed experimentally, mainly because of the damping of the flow velocity near the walls of the microchannels for pressure-driven flows. Because the flow that drives the vortex at the opening of the diamondshaped cavity is near the $30-\mu \mathrm{m}$-wide channel wall, the maximum fluid rotational velocity inside the cavity in Fig. 1b, c is only about $20 \%$ of the average flow velocity in the channel.

To attain ultrahigh rotational velocities in the microvortex, we designed a trapezoidal microchamber connected to a constricted microchannel that was placed asymmetrically along the width of a $200-\mu \mathrm{m} \times 200-\mu \mathrm{m}$ channel (see supplementary information). Using this design, we measured maximum rotational velocities as high as $12 \mathrm{~m} \mathrm{~s}^{-1}$ at a distance of $10 \mu \mathrm{m}$ from the vortex core, which corresponds to a radial acceleration of $1.4 \times 10^{7} \mathrm{~m} \mathrm{~s}^{-2}$ or $1.4 \times 10^{6} \mathrm{~g}$ (Fig. 1d). We measured rotation rates (Fig. $1 \mathrm{~d}$ ), both by the time taken for a photolysed (uncaged) plug of fluorescein dye to travel a calibrated distance $(5 \mu \mathrm{m})$ from a focused nitrogen photolysis laser beam to an argon laser probe volume (Fig. 1d, squares), and by the time taken for the plug of dye to travel completely around the microvortex (Fig. 1d, open circles) ${ }^{7}$ (and see supplementary information).

The ability to use fluid flow with no moving parts to generate large centrifugal forces should prove useful for integrated microanalytical separations - for example, in sample preparation.

\section{J. Patrick Shelby, David S.W. Lim,}

Jason S. Kuo, Daniel T. Chiu

Department of Chemistry, University of Washington, Seattle, Washington 98195-1700, USA

e-mail: chiu@chem.washington.edu

1. Reyes, D. R., Iossifidis, D., Auroux, P.-A. \& Manz, A. Anal. Chem 74, 2623-2636 (2002).

2. Auroux, P.-A., Iossifidis, D., Reyes, D. R. \& Manz, A. Anal. Chem. 74, 2637-2652 (2002).

3. Thorsen, T., Maerkl, S. J. \& Quake, S. R. Science 298, 580-584 (2002).

4. Beebe, D. J., Mensing, G. A. \& Walker, G. M. Annu. Rev. Biomed. Eng. 4, 261-286 (2002).

5. Ng, J. M. K., Gitlin, I., Stroock, A. D. \& Whitesides, G. M. Electrophoresis 23, 3461-3473 (2002).

6. Shankar, P. N. \& Deshpande, M. D. Annu. Rev. Fluid Mech. 32, 93-136 (2000).

7. Shelby, J. P. \& Chiu, D. T. Anal. Chem. 75, 1387-1392 (2003). Supplementary information accompanies this communication on Nature's website.

Competing financial interests: declared none. 